\title{
The economic cost of implementing antigen-based rapid diagnostic tests for COVID-19 screening in high-risk transmission settings: evidence from Germany
}

Alfonso Valenzuela Hurtado ${ }^{7^{*}}$ (D), Hoa Thi Nguyen ${ }^{1,2}$, Viktoria Schenkel ${ }^{3}$, Jonas Wachinger ${ }^{1}$, Joachim Seybold ${ }^{3 \dagger}$, Claudia M. Denkinger ${ }^{2+}$ and Manuela De Allegri ${ }^{1+}$

\begin{abstract}
Background: Antigen-based rapid diagnostic tests (Ag-RDT) have been implemented in hospitals and nursing homes to screen for infectious individuals without symptoms suggestive of SARS-CoV-2 infections and to prevent entry into these high-risk settings. Despite their benefits for screening, the cost of large-scale implementation is largely understudied. Our study presents evidence on their implementation costs in high-risk settings. This study aimed to estimate the economic costs of implementing Ag-RDT-based screening for SARS-CoV-2 in two tertiary care hospitals (University Hospital Heidelberg - UKHD, and Charité - Universitätsmedizin Berlin) and one nursing home in Germany.

Methods: We adopted a health system perspective and followed the three sequential steps to costing: identification of resources, measurement of resource consumption, and valuation of costs. Data on resource consumption were collected between October 2020 and April 2021 through various techniques and data sources. The cost estimation considered all costs along the screening algorithm including PCR confirmation tests for positive cases. We estimated the costs for the two implementation modalities observed: staff dedicated exclusively to screening and staff not dedicated exclusively to screening. Furthermore, cost estimations were performed under both observed capacity use and hypothetical capacity use assumptions (60, 80 and 100\%).
\end{abstract}

\footnotetext{
* Correspondence: alfonso.valenzuela.hurtado@uni-heidelberg.de

${ }^{\dagger}$ Joachim Seybold, Claudia M. Denkinger and Manuela De Allegri are contributed equally to this work.

${ }^{1}$ Heidelberg Institute of Global Health, Heidelberg University Hospital and Faculty of Medicine, Heidelberg University, Heidelberg, Germany Full list of author information is available at the end of the article
}

(c) The Author(s). 2022 Open Access This article is licensed under a Creative Commons Attribution 4.0 International License, which permits use, sharing, adaptation, distribution and reproduction in any medium or format, as long as you give appropriate credit to the original author(s) and the source, provide a link to the Creative Commons licence, and indicate if changes were made. The images or other third party material in this article are included in the article's Creative Commons licence, unless indicated otherwise in a credit line to the material. If material is not included in the article's Creative Commons licence and your intended use is not permitted by statutory regulation or exceeds the permitted use, you will need to obtain permission directly from the copyright holder. To view a copy of this licence, visit http://creativecommons.org/licenses/by/4.0/ The Creative Commons Public Domain Dedication waiver (http://creativecommons.org/publicdomain/zero/1.0/) applies to the data made available in this article, unless otherwise stated in a credit line to the data. 
Results: Our study indicates that the average cost per Ag-RDT is highly dependent on the capacity use and implementation mode. Staff time and test kits are the two main cost drivers of implementing the large-scale screening programs for SARS-CoV-2 using Ag-RDTs. For hospitals, the average cost per test in UKHD was $€ 30.12$ (capacity observed); €14.56 (non-dedicated mode); €19.47, €16.37, €14.53 at 60, 80, 100\% capacity respectively (dedicated mode); and at Charité €13.10 (non-dedicated mode). For the nursing home the estimated average cost per test was $€ 15.03$ (non-dedicated mode).

Conclusions: The information on the estimated costs by mode of implementation and capacity use may support the planning of Ag-RDT-based covid-19 screening programs suitable for each institution. Further research is needed to cost this screening strategy for COVID-19 in other high-risk, high-income settings to reach generalizability.

Keywords: Antigen-based rapid diagnostic test, SARS-CoV-2, Screening, Implementation costs, Germany

\section{Introduction}

Despite increasing vaccination rates for SARS-CoV-2, screening of asymptomatic individuals remains crucial, as they may account for up to $45 \%$ of all SARS-CoV- 19 infections [1]. This is particularly important upon entry in settings with persons that are at high risk of severe COVID-19 infection, such as hospitals and nursing homes, where it is critical to avoid exposure to the virus and thereby avoid new infections [2, 3]. However, scaling-up screening has become a major challenge during this pandemic [4].

Among the different SARS-CoV-2 diagnostic tests approved by the WHO and national health authorities, the gold standard is the reverse transcriptase-polymerase chain reaction (RT-PCR) [5]. However, the turn-around time of large throughput RT-PCR often renders it ineffective for rapid detection [3], mainly in high-risk settings (e.g. hospitals or nursing homes), where screening for asymptomatic individuals is key to deciding on access and thus minimizing the exposure to the virus of the population at risk. Consequently, everyday practice requires complementary diagnostic alternatives to detect infectious individuals [6]. An easy-to-use, rapid point of care $(\mathrm{PoC})$ test can be used to expand screening capacity [7]. SARS-CoV-2 antigen-based rapid detection tests (Ag-RDTs) offer good accuracy, rapid PoC detection of infectious individuals and ease of use [7]. Therefore, implementation of Ag-RTDs for screening asymptomatic individuals has occurred widely in Germany in nursing homes and to some extent in hospitals. However, no published data exist on the economic costs of the implementation of Ag-RDT-based screening programs for COVID-19 in high-risk settings.

In the absence of evidence on the costs of screening asymptomatic people with Ag-RDTs in high-risk settings, and in order to inform the roll-out of Ag-RDTbased screening programs, we estimated the costs of implementing Ag-RDT-based screening programs for asymptomatic persons in high-risk settings. Given the continued preponderance of the pandemic, our ambition has been that of generating a sound evidence-base to guide further implementation of screening in high-risk settings, considering the full economic cost of the program beyond only the cost of the single test kit. Specifically, we estimated the costs in two university hospitals and one nursing home in Germany and addressed three objectives: i) estimate the average cost per test implemented in hospital settings; ii) project the estimated cost for patient screening using Ag-RDTs in hospitals with different patient volumes; iii) estimate the average cost per test implemented in nursing homes.

\section{Methods}

\section{Study setting and intervention}

This study was carried out in two tertiary hospitals in Germany: Heidelberg University Hospital (UKHD) and Charité - Universitätsmedizin Berlin (Charité). UKHD and Charité are two of the largest tertiary hospitals in Germany, with 1991 [8] and 3001 [9] beds, respectively. Both institutions have large laboratory services capacity for patient diagnosis (including RT-PCR testing); however, laboratory capacity has been constrained due to increased demand since the onset of the current pandemic. Therefore, UKHD implemented Ag-RDTs for the screening of patients presenting for elective procedures without symptoms suggestive of SARS-CoV-2 (including day clinics) prior to admission in all clinics starting from October 2020, either by dedicated staff (staff already employed in the facility who are redirected to work exclusively on Ag-RTDs patient screening), or by non-dedicated staff (staff already employed in the facility who perform Ag-RDTs only when required within the framework of their daily work routine). Ag-RDTs at Charité were implemented for the screening of patients presenting for elective procedures without symptoms suggestive of SARS-CoV-2 in the neurology outpatient department from March to April 2021, using the nondedicated staff mode only for the purpose of this study. Both hospitals used the Standard Q COVID-19 Ag kit (SD Biosensor, Republic of Korea) for patient screening 
due to its high sensitivity (76.6\%) and specificity (99.3\%) in persons with high viral load infections $(\mathrm{CT}<25)$ [10]. In patients with a positive Ag-RDT, an additional nasopharyngeal swab was collected for confirmatory RT-PCR [11].

Separately, we assessed costs in a nursing home in the Rhein-Neckar-Kreis, an administrative district in the South of Germany. In this setting, screening was carried out only with non-dedicated staff for residents, visitors and health workers. The two implementation mode categories, dedicated staff and non-dedicated staff, reflect the different ways in which staff were engaged to carry out rapid testing in each of the settings, with different implications in terms of opportunity cost. In other words, dedicated staff is exclusively assigned to implement Ag-RDTs, and when the number of patients tested is lower than the planned testing volume, their time is unused and thus the opportunity cost of their time increase. Meanwhile, non-dedicated staff performs AgRDT testing alongside with their routine nursing tasks, thus we assumed that their time is used at $100 \%$ capacity and accordingly, the opportunity cost of their time is not affected by varying testing volume.

\section{Study design}

This costing study adopted a health system perspective to provide relevant evidence to decision makers on the economic cost of implementing Ag-RDTs. Data on resource consumption were collected from all study sites between October 2020 and April 2021.

In line with the study objectives, we followed the bottom-up micro-costing approach [12] to estimate the cost of implementing Ag-RTDs in the two hospitals (study objective 1) and the nursing home (study objective 3). To project the costs associated with patient screening using Ag-RDTs in hospitals of different size in Germany (study objective 2), we compiled the average cost estimates obtained in study objective 1 with data on service volume for hospitals of different patient volume from open data sources.

\section{Costing procedures and data sources}

Our micro-costing approach unfolded over three sequential steps. First, we identified which resources were necessary for the implementation of Ag-RDTs based on standard operating procedures (SOPs) and direct observation (only in hospitals). Subsequently, we classified the identified items in recurrent costs (staff, test kits and consumables, protective gear, and variable overheads) and capital costs (fix overheads, building, and equipment).

Second, we measured the consumption for the identified resources using different techniques and data sources. Table 1 presents an overview of the methods and data sources for the measurement of resource consumption and the valuation of costs used in all study settings. For recurrent costs, given that staff time to implement a test can vary, we conducted direct observation at UKHD and Charité to record the average time employed by staff to complete the different steps to perform a test, including patient registration, sample taking, test implementation, results monitoring and documentation. Since direct observation at the nursing home was not possible, we assumed that staff time consumption would be equivalent to what was observed at UKHD and Charité. We measured the resource consumption of test kits and other recurrent cost items using the corresponding SOPs for Ag-RDTs implementation from the respective study sites. The data from UKHD was utilized for the estimations of the nursing home.

Third, to value costs of resource consumption we applied both a bottom-up and a top-down approach (Table 1). For recurrent costs, we valued staff cost considering the two different implementation modes, i.e., dedicated vs. nondedicated staff. Specifically, for the non-dedicated staff mode of implementation (implemented in Charité, in certain UKHD clinics, and the nursing home), we estimated staff cost by multiplying the average time in minutes needed to perform one test by the staff cost per minute. For the dedicated staff mode (implemented in other UKHD clinics), we estimated the staff cost per test by dividing the daily staff cost by the number of tests performed daily. Since nurses carry out most tests in our study settings, we used the average cost of nurses to value health staff costs, which include gross salaries and costs to the employer. In addition, we added the corresponding cost of training to the staff cost per test. Given that test kits contain all the necessary materials to carry out one test, we used the purchase price of the test kit to value the cost of all test materials. Moreover, we added the cost of the consumables required per test (hand sanitizing solution and tissues). We valued the costs of protective gear by multiplying the quantities of single-use items and the estimated use of multipleuse items per test by the corresponding unit cost (bottomup approach), obtained either from market prices for UKHD or internal information for Charité (Table 1). We valued variable overhead using estimates of hospital cost data in Germany from government statistics for UKHD and the nursing home and internal cost information for Charité.

To value capital costs, we used both a top-down and bottom-up approach (Table 1). For UKHD we mostly used market prices, while for Charité we used internal information provided by the purchasing and human resources departments of the hospital. Fix overhead cost information was obtained from different sources including internal purchasing price and open data sources. We estimated the building cost by multiplying the room space used by the commercial rent prices per square meter for UKHD and the nursing home and internal 


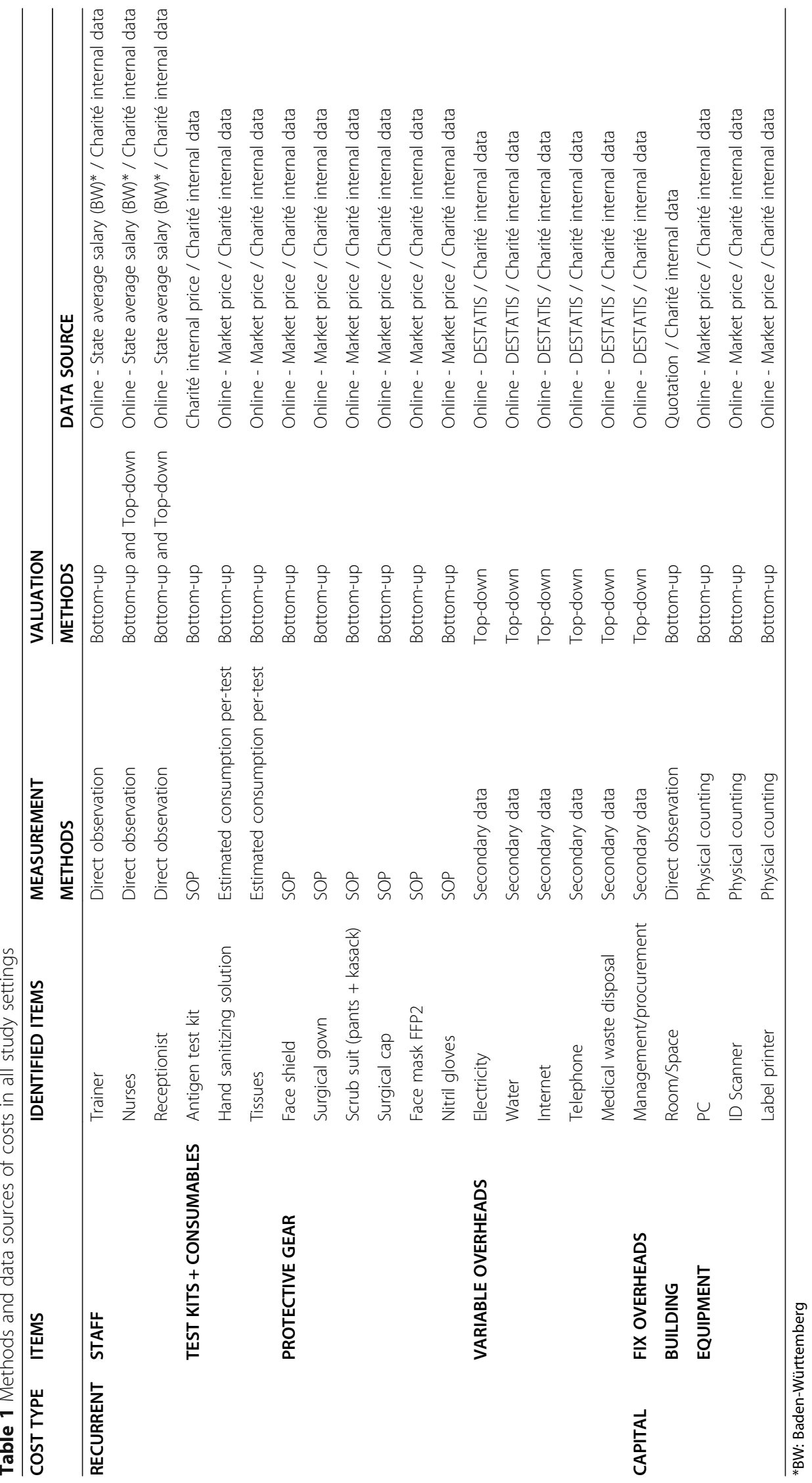


information for Charité. For both hospitals, we computed equipment costs using information on quantities, unit prices, and useful years of life.

\section{Cost analysis}

Data were analyzed in Microsoft Excel 2016. Costs were calculated in Euros using 2021 as the base year. We computed the average cost per test separately for UKHD, Charité, and the nursing home and for the two implementation modalities (i.e., dedicated and nondedicated staff). Under the non-dedicated staff mode, our calculation assumes that the staff time is used at maximum capacity (100\%) across all tasks, not screening alone. Our assumption is based on an understanding that nursing staff divide their time across a number of activities, with Ag-RDTs screening being only one of them. Given that for the dedicated staff mode of implementation, the number of tests performed per day depends not only on the staff time allocated to testing, but also the volume of patients requiring tests, we calculated the average cost per test for the observed capacity use and at three different capacity use assumptions, specifically at $60,80,100 \%$. Moreover, we included the RT-PCR confirmation cost for positive cases in the average cost per test. For the calculation of RT-PCR confirmation costs, we used an incidence rate of 0.001 , the sensitivity (76.6\%) and specificity (99.3\%) of the above-mentioned Ag-RDT [10], and the reimbursement rate of RT-PCR in Germany as of February 2021(€50.50) [13].

Related cost projection of Ag-RDTs for hospitals of different service volume was calculated using the estimate of the average costs per test obtained from UKHD and Charite and the expected service volume for each hospital category. Specifically, we used the available open data sources $[8,14,15]$ and classified hospitals into three categories based on the number of beds: small $(0-300)$, medium (301-500), and large $(500+)$. For each hospital category, we used the number of daily in-patient admissions to project the screening costs $[8,14,15]$.

\section{Sensitivity analysis}

To assess uncertainty around the average cost per test in each setting, we conducted sensitivity analyses using the minimum and maximum values of the two important and variable cost items: staff time to perform one test $(8-16 \mathrm{~min})$ and the unit cost of a test kit $(€ 4-€ 6.90)$.

\section{Ethics}

The costing study received ethical approval (S-802/2020) from the Ethics Commission of the Medical Faculty of Heidelberg University.

\section{Results}

In line with our objectives, we first report the cost estimates for the two hospitals (Table 2 and Table 3 ) and their cost structure (Fig. 1), then the projection of the cost estimates in hospitals of different patient volume (Fig. 2), and finally the cost estimates for the nursing home (Table 4).

We report the average cost per test implemented at UKHD in Table 2 and at Charité in Table 3. For the non-dedicated mode of implementation, where we assume the maximum staff capacity $(100 \%)$, the estimated average costs per test implemented at UKHD were $€ 14.56$ and $€ 13.10$ at Charité (including PCR confirmation). The sensitivity analysis for UKHD showed that the average cost per test can vary between $€ 11.06$ and $€ 18.34$, when implementation time varies from 8 to 16

Table 2 The estimated average cost per test of Ag-RDT implementation in UKHD

\begin{tabular}{|c|c|c|c|c|c|c|}
\hline \multirow[t]{3}{*}{ SCENARIO } & \multirow[t]{3}{*}{ COST ITEMS } & \multicolumn{4}{|c|}{ DEDICATED MODE } & \multirow{3}{*}{$\begin{array}{l}\text { NON-DEDICATED } \\
\text { MODE }\end{array}$} \\
\hline & & \multirow{2}{*}{$\begin{array}{l}\text { CAPACITY } \\
\text { OBSERVED }\end{array}$} & \multicolumn{3}{|c|}{ HYPOTHETICAL ASSUMPTION } & \\
\hline & & & $60 \%$ & $80 \%$ & $100 \%$ & \\
\hline \multirow[t]{7}{*}{ UKHD } & Protective gear & $€ 0.68$ & $€ 0.53$ & $€ 0.48$ & $€ 0.46$ & $€ 0.47$ \\
\hline & Test + consumables & $€ 5.58$ & $€ 5.58$ & $€ 5.58$ & $€ 5.58$ & $€ 5.58$ \\
\hline & Staff & $€ 20.12$ & $€ 10.95$ & $€ 8.21$ & $€ 6.56$ & $€ 6.56$ \\
\hline & Overhead - variable & $€ 0.64$ & $€ 0.34$ & $€ 0.29$ & $€ 0.26$ & $€ 0.27$ \\
\hline & Overhead - fix & $€ 2.37$ & $€ 1.53$ & $€ 1.30$ & $€ 1.18$ & $€ 1.19$ \\
\hline & Building & $€ 0.31$ & $€ 0.14$ & $€ 0.11$ & $€ 0.08$ & $€ 0.09$ \\
\hline & Equipment & $€ 0.04$ & $€ 0.02$ & $€ 0.01$ & $€ 0.01$ & $€ 0.01$ \\
\hline \multicolumn{2}{|c|}{ AVERAGE COST PER TEST } & $€ 29.73$ & $€ 19.08$ & $€ 15.98$ & $€ 14.14$ & $€ 14.17$ \\
\hline \multicolumn{2}{|c|}{ (+) PCR CONFIRMATION } & $€ 30.12$ & $€ 19.47$ & $€ 16.37$ & $€ 14.53$ & $€ 14.56$ \\
\hline \multirow{2}{*}{\multicolumn{2}{|c|}{ SENSITIVITY ANALYSIS }} & LOW & $€ 14.40$ & $€ 12.21$ & $€ 11.02$ & $€ 11.06$ \\
\hline & & $\mathrm{HIGH}$ & $€ 24.45$ & $€ 20.45$ & $€ 18.30$ & $€ 18.34$ \\
\hline \multicolumn{2}{|c|}{ Staff time per-test: } & \multicolumn{5}{|c|}{ Minimum $8 \mathrm{~min}$, average $12 \mathrm{~min}$, maximum $16 \mathrm{~min}$} \\
\hline
\end{tabular}


Table 3 The estimated average cost per test of Ag-RDT implementation in Charité Berlin

\begin{tabular}{lll}
\hline SCENARIO & COST ITEMS & NON-DEDICATED MODE \\
& & $\mathbf{1 0 0 \%}$ \\
\hline CHARITÉ & Protective gear & $€ 0.27$ \\
& Test + consumables & $€ 5.53$ \\
& Staff & $€ 5.22$ \\
& Overhead variable & $€ 0.06$ \\
& Overhead fix & $€ 1.44$ \\
& Building & $€ 0.17$ \\
AVERAGE COST PER TEST & Equipment & $€ 0.02$ \\
$(+)$ PCR confirmation & & $€ 12.71$ \\
SENSITIVITY ANALYSIS & & $€ 13.10$ \\
& LOW & $€ 9.91$ \\
Staff time per-test: & HIGH & $€ 16.28$
\end{tabular}

min and the cost of the above-mentioned test kit varies from $€ 4$ to $€ 6.90$ (Table 1 ). The sensitivity analysis for Charité showed a variability of the average cost between $€ 9.91$ and $€ 16.28$ (Table 3) with the same parameter ranges. For the dedicated mode of implementation, applicable to UKHD only, the estimated average cost per test ranged from $€ 30.12$ per test under the observed capacity use to $€ 14.53$ (including PCR confirmation) per test under the $100 \%$ capacity use assumption (Table 2 ).

The cost structure is visualized in Fig. 1, which shows that the main cost drivers in hospitals were staff salaries and test kits. Together they accounted for an average of $85 \%$ of the total cost, while all recurrent costs accounted for $89 \%$ of the total cost (non-dedicated mode).

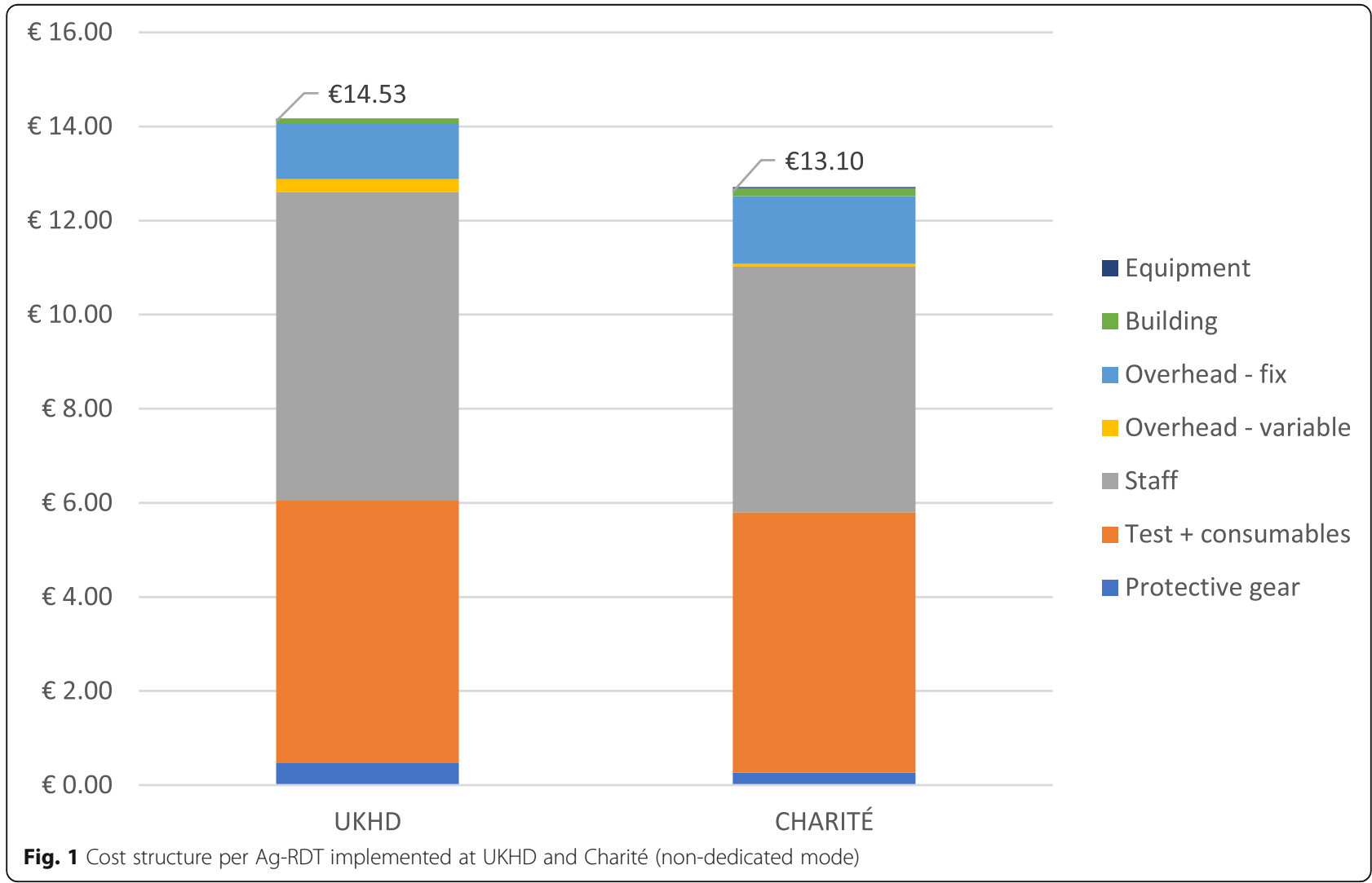




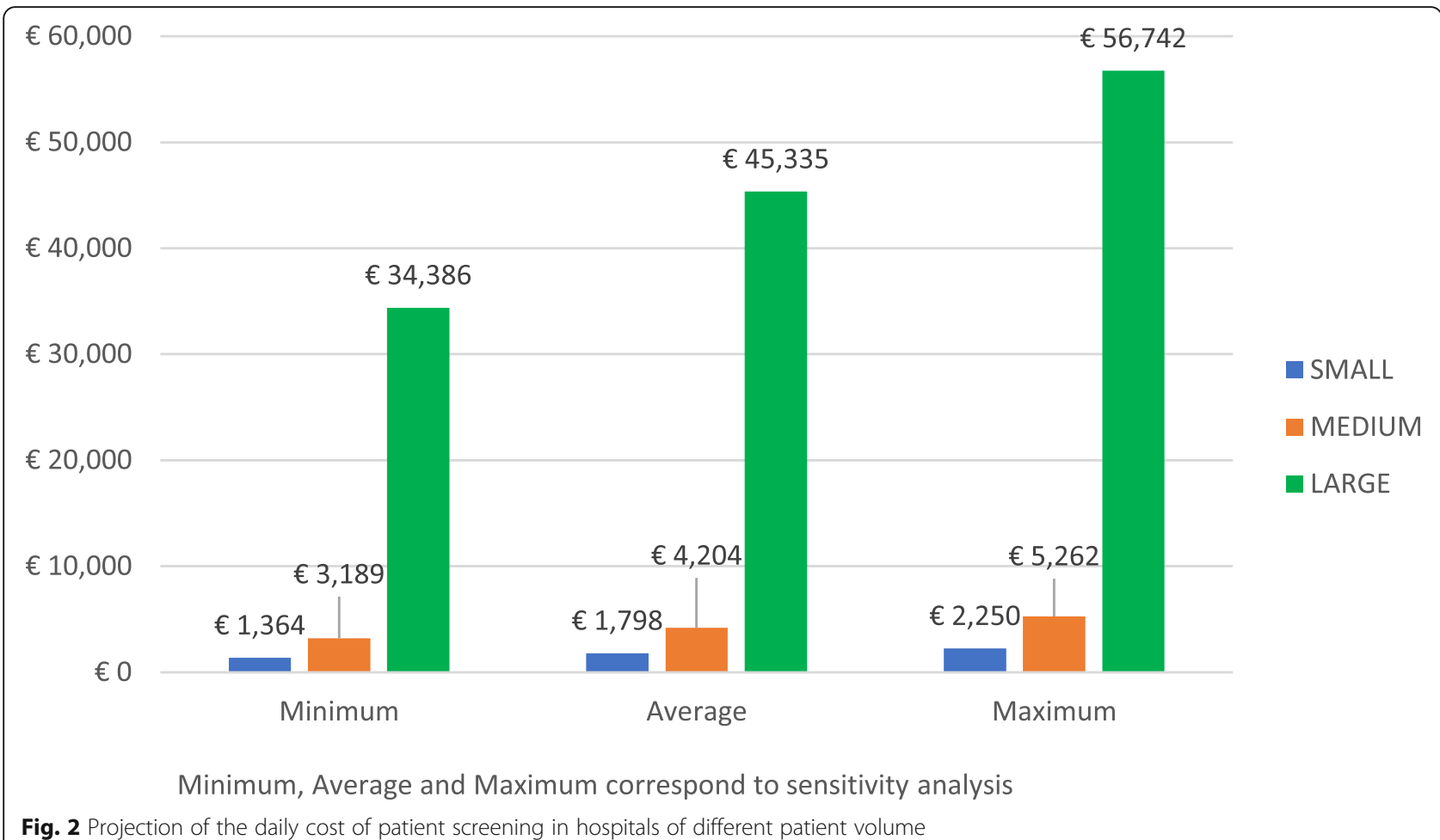

Figure 2 displays findings from the cost projection in hospitals of different patient volume. Screening of electively admitted patients would cost $€ 1.798$, $€ 4.204$, $€ 45.335$ a day in small, medium, and large hospitals respectively. A sensitivity analysis modifying the two main cost drivers, staff time and cost of tests kits, led to ranges from $€ 1.364$ to $€ 2.250$ for the small hospital, from $€ 3.189$ to $€ 5.262$ for the medium hospital, and from $€ 34.386$ to $€ 56.742$ for the large hospital.
In the nursing home, at maximum capacity utilization, the average costs per test would be $€ 15.17$ (including PCR confirmation costs). The sensitivity analysis modifying the two main cost drivers led to ranges from $€ 11.80$ to $€ 18.82$. As with hospitals, the main cost drivers in the nursing home were staff time and test kits cost, as together they represented $82 \%$ of the total cost. Recurrent costs accounted for $90 \%$ of total costs.

Our results indicate that in both the dedicated staff mode and the non-dedicated staff mode the estimated

Table 4 Estimated average cost per test at the nursing home

\begin{tabular}{lll}
\hline SCENARIO & COST ITEMS & NON-DEDICATED MODE \\
\hline NURSING HOME & Protective gear & $\mathbf{1 0 0 \%}$ \\
& Test + consumables & $€ 0.90$ \\
& Staff & $€ 5.58$ \\
& Overhead - variable & $€ 6.56$ \\
& Overhead - fix & $€ 0.26$ \\
& Building / space & $€ 1.17$ \\
& Equipment & $€ 0.28$ \\
AVERAGE COST PER TEST & & $€ 0.03$ \\
$(+)$ PCR confirmation & & $€ 14.78$ \\
SENSITIVITY ANALYSIS & LOW & $€ 15.17$ \\
Staff time per-test: & HIGH & $€ 11.80$ \\
& & Minimum 8 min, average 12 min, maximum 16 min
\end{tabular}


cost per test implemented decreases as the maximum capacity utilization is reached.

\section{Discussion}

To the best of our knowledge, this costing study represents the first attempt to estimate the economic costs associated with implementing Ag-RDTs for screening of SARS-CoV-2 in a high-risk setting. Our estimates and projections are intended to provide initial evidence to inform decisions on planning and implementing similar screening strategies in the future. Given limited implementation experience on PoC testing in high-risk settings and related evidence on costing, our findings may also be useful when considering the introduction of test screening programs to prevent outbreaks of other airborne diseases, such as influenza in high-risk settings. Furthermore, the presented methods could be replicated in other high-income settings, with the possibility of estimating the cost of universal screening, including healthcare and administrative staff and visitors.

Our estimates show that the average cost per test implemented by non-dedicated staff at full capacity $(100 \%)$ was $€ 14.56$ in UKHD, $€ 13.10$ in Charité and $€ 15.17$ in the nursing home. The differences in costs are small and are mainly due to differences in the salaries of nurses and to a lesser extent to differences in the costs obtained for the other items. As can be seen in Fig. 2, in both hospitals staff time and test kits account for approximately $85 \%$ of the cost per test implemented. Similarly, the cost per test implemented in the nursing home is highly dependent on the same cost drivers.

According to our estimates, the cost is primarily driven by staff time, therefore the cost of antigen testing is highly dependent on choosing the optimal implementation modality that optimizes staff capacity use. However, this is complex, as it requires detailed planning across different hospital departments and adaptation of patient flow. Estimates of the non-dedicated approach assume that staff works at full capacity, performing tasks pertaining to screening as well as many other nursing tasks, while those of the dedicated approach are based on the observed capacity use of staff time. An important cost difference was seen between clinics that implemented both modalities. Implementing Ag-RDTs at UKHD with dedicated staff $(€ 30.12)$ was more than twofold than implementing them with non-dedicated staff $(€ 14.56)$ (Table 1). The difference was driven by the fact that at the time of the study, screening demand, defined in relation to the number of people presenting at UKHD with need to be tested, was not so high as to lead dedicated staff to work at full capacity. This increased the opportunity cost of staff time due to unused time of nurses who were assigned exclusively to do Ag-RDTs testing, possibly leading to efficiency losses in the management of nursing staff time. This points to the importance of adopting the appropriate implementation modality, taking into account the time constraints of health care workers on one side and the expected volume of service on the other, to maximize efficient use of human resources. Considering that the implementation of the dedicated staff mode also implies removing staff temporarily from other nursing tasks, this modality should be considered if, through proper planning, the expected service volume is close to maximum utilization. When this is not feasible, non-dedicated staff mode may represent a preferable option. It is important to note, however, that the time it takes for non-dedicated staff to carry out the tests is time taken away from other nursing activities, capturing the opportunity cost of staff time in performing the tests. Hence, selecting either implementation mode should be a contextual decision, considering a facility capacity, expected testing volume, and overall management feasibility. The second cost driver is the cost of test kits, which have recently dropped rapidly as the quantity demanded increased and a myriad of new tests, although of different accuracy and quality, are now easily available.

Due to daily fluctuations in the number of patients attending clinics, we observed a utilization of the screening capacity use of approximately $45 \%$ in clinics that adopted the dedicated-staff approach. Returning to a concept of opportunity cost of staff time outlined earlier, this is because there is downtime throughout the day that is not used to perform other functions, when the staff is assigned exclusively to carry out the tests during their working shift. Dedicated staff mode is more prone to lower efficiency and higher cost in relation to the non-dedicated mode when the planning for the maximum use of staff time is not guaranteed.

We consider that a strength of our study is the evidence on the costs of implementing PoC testing for SARS-CoV-2 detection in high-risk settings, as well as the replicability of the presented methodology.

\section{Methodological considerations}

We need to acknowledge the limited generalizability that arises from our small sample of only two tertiary care hospitals and one nursing home, all located in Germany. The issues of accessibility during the pandemic limited our capacity to enlarge our sample to other high-risk settings that perform screening in Germany and other countries. However, the fact that estimates were relatively stable across settings and across the modifications applied in the sensitivity analysis, such as those related to service volume, suggests that generalizability within the German setting is not as limited as the small study sample would imply. We need to note that by adjusting for different costs for the testing kits, our sensitivity 
analysis also accounts for different procurement costs since these costs were categorized as fix overheads and calculated as a percentage of the total costs.

Additionally, we need to note that the projections are based exclusively on estimates derived from large tertiary care hospitals even when made for medium- and lowvolume facilities. While the sample size is limited, the hospitals are likely to reflect the cost of staff time and implementation complexity for other hospitals. Differences in possible purchase cost across larger volumes were captured with sensitivity analyzes. To account for the potential bias that may arise, we have performed an analysis considering different capacity assumptions. Moreover, due to the risks of conducting observations in nursing homes, the cost estimates were derived exclusively from SOPs and the hospital estimates.

We also need to note two potential limitations of our cost computation, one related to potential costs induced by outbreaks caused by false negative test results and the other related to the cost of having to repeat some tests twice due to the test failure rate. In the first instance, we recognize this as a valid concern, but simply beyond the scope of our analysis, which does not intent to relate cost estimates to transmission parameters. It should be noted, however, that no outbreaks were observed during the implementation period. In the second instance, while in principle, we would have needed to adjust cost estimates to account for test failure rate, we did not because prior evidence revealed that the test implemented in our study, Standard Q COVID-19 Ag SD Biosensor, had no invalid results [16].

\section{Conclusions}

It is important to estimate the volume of tests expected to be performed in the short term in order to choose the appropriate mode of implementation, and thus make the best possible use of available resources. In this way each facility can determine, according to its expected capacity use, the most appropriate screening program for SARSCoV-2 that can cover not only patients, but also health personnel, administrative staff, and visitors. More evidence is needed on the costs of Ag-RDT implementation in other high-risk, high-income settings, especially in medium and small hospitals, to enhance the evidencebase for decision making.

\section{Abbreviations}

Ag-RDT: Antigen-based Rapid Diagnostic Test; RT-PCR: Reverse Transcriptase Polymerase Chain Reaction; PoC: Point of Care; UKHD: Universitätsklinikum Heidelberg; SOP: Standard Operating Procedure

\section{Acknowledgements}

we are grateful to Britta Knorr, Annette Moehlenbruch, Steffen Till, Michael Denkinger for contribute SOPs from nursing homes. And to Benedikt Fischer for data collection. We also wish to thank Lisa Krueger, and Christoph Schulz for facilitating access to the antigen test study site for the costing.

\section{Authors' contributions}

MDA, JS, and CMD conceptualized the study. AH and HN analyzed the data, supported by JW, CMD, JS, VS, and MDA. MDA, AH, HN and CMD drafted the manuscript. All co-authors critically revised and approved the final version of the manuscript.

\section{Funding}

This study was funded partially by the Ministry of Science, Research and the Arts of Baden-Württemberg, Germany, as well as UKHD internal funds, internal funds of the Heidelberg Institute of Global Health, CONACYT-DAAD and the German Ministry of Education and Research through Forschungsnetzwerk der Universitätsmedizin zu COVID-19, (B-FAST). Open Access funding enabled and organized by Projekt DEAL.

\section{Availability of data and materials}

All data generated or analysed during this study are included in this published article.

\section{Declarations}

\section{Ethics approval and consent to participate}

The study received ethical approval (S-802/2020) from the Ethics Commission of the Medical Faculty of Heidelberg University. Since we collected no information from human subjects, no informed consent was required.

\section{Consent for publication}

Not applicable.

\section{Competing interests}

The authors declare that they have no competing interests.

\section{Author details}

${ }^{1}$ Heidelberg Institute of Global Health, Heidelberg University Hospital and Faculty of Medicine, Heidelberg University, Heidelberg, Germany. ${ }^{2}$ Division of Clinical Tropical Medicine, Heidelberg University Hospital and Faculty of Medicine, Heidelberg University, Heidelberg, Germany. ${ }^{3}$ Charité -

Universitätsmedizin Berlin, corporate member of Freie Universität Berlin, Humboldt-Universität zu Berlin, and Berlin Institute of Health; Medical Directorate, Berlin, Germany.

Received: 27 October 2021 Accepted: 4 February 2022 Published online: 14 February 2022

\section{References}

1. Oran DP, Topol EJ. Prevalence of asymptomatic SARS-CoV-2 infection. Ann Intern Med. 2020;173(5):362-7. https://doi.org/10.7326/M20-3012.

2. Viswanathan M, Kahwati L, Jahn B, Giger K, Dobrescu Al, Hill C, et al. Universal screening for SARS-CoV-2 infection: a rapid review. Cochrane Database Syst Rev. 2020;9(9). https://doi.org/10.1002/14651858.CD013718.

3. Mina MJ, Andersen KG. COVID-19 testing: one size does not fit all. Science. 2021;371(6525):126-7. https://doi.org/10.1126/science.abe9187.

4. Peto T. COVID-19: Rapid Antigen detection for SARS-CoV-2 by lateral flow assay: a national systematic evaluation for mass-testing. medRxiv. 2021:2021. 01.13.21249563.

5. Tahamtan A, Ardebili A. Real-time RT-PCR in COVID-19 detection: issues affecting the results. Expert Rev Mol Diagn. 2020;20(5):453-4. https://doi. org/10.1080/14737159.2020.1757437.

6. Mina MJ, Peto TE, García-Fiñana M, Semple MG, Buchan IE. Clarifying the evidence on SARS-CoV-2 antigen rapid tests in public health responses to COVID-19. Lancet. 2021;397(10283):1425-7. https://doi.org/10.1016/S01406736(21)00425-6.

7. Mina MJ, Parker R, Larremore DB. Rethinking Covid-19 test sensitivity - a strategy for containment. N Engl J Med. 2020;383(22):e120. https://doi.org/1 0.1056/NEJMp2025631.

8. Zahlen Daten Fakten UKHD 2018 [press release]. Universität Heidelberg, Dezember 20192019.

9. Einblicke 2020 Jahresbericht [press release]. Berlin: Geschäftsbereich Unternehmenskommunikation, April 20212021.

10. Krüger LJ, Gaeddert M, Köppel L, Brümmer LE, Gottschalk C, Miranda IB, et al. Evaluation of the accuracy, ease of use and limit of detection of novel, 
rapid, antigen-detecting point-of-care diagnostics for $<$ em $>$ SARS-CoV-2</ em\&gt. medRxiv. 2020:2020.10.01.20203836.

11. Wachinger J, Olaru ID, Horner S, Schnitzler P, Heeg K, Denkinger CM. The potential of SARS-CoV-2 antigen-detection tests in the screening of asymptomatic persons. Clin Microbiol Infect. 2021:S1198-743X(21)00412-2.

12. Drummond MF, Sculpher MJ, Claxton K, Stoddart GL, Torrance GW.

Methods for the economic evaluation of health care Programmes. Oxford: Oxford: Oxford University Press; 2015.

13. Gesundheit Bf. Verordnung zum Anspruch auf Testung in Bezug auf einen direkten Erregernachweis des Coronavirus SARS-CoV-2 (CoronavirusTestverordnung - TestV). In: Gesundheit Bf, editor. Bonn2021.

14. (G-BA) GB. Referenz bericht 2018 Waldkliniken Eisenberg 2019.

15. (G-BA) GB. Referenzbericht 2016 Bundeswehrkrankenhaus UIm. 2017:13.

16. Krüger $L$, Tanuri A, Lindner AK, Gaeddert M, Köppel L, Tobian F, et al. Accuracy and ease-of-use of seven point-of-care SARS-CoV-2 antigendetecting tests: A multi-centre clinical evaluation. EBioMedicine. 2021;75: 103774. doi:https://doi.org/10.1016/j.ebiom.2021.103774. [published online ahead of print, 2021 Dec 24].

\section{Publisher's Note}

Springer Nature remains neutral with regard to jurisdictional claims in published maps and institutional affiliations.

Ready to submit your research? Choose BMC and benefit from:

- fast, convenient online submission

- thorough peer review by experienced researchers in your field

- rapid publication on acceptance

- support for research data, including large and complex data types

- gold Open Access which fosters wider collaboration and increased citations

- maximum visibility for your research: over $100 \mathrm{M}$ website views per year

At BMC, research is always in progress.

Learn more biomedcentral.com/submissions 